\section{FDA Guidance for Industry' Dissolution Testing of Immediate Release Solid Oral Dosage Forms}

This guidance is developed for immediate release (IR) dosage forms and is intended to provide (I) general recommendations for dissolution testing; (2) approaches for setting dissolution specifications related to the biopharmaceutic characteristics of the drug substance; (3) statistical metbods for comparing dissolution profiles; and (4) a process to belp determine when dissolution testing is sufficient to grant a waiver for an in vivo bioequivalence study. This document also provides recommendations for dissolution tests to belp ensure continuous drug product quality and performance after certain postapproval mamufacturing changes. Summary information on dissolution methodology, apparatus, and operating conditions for dissolution testing of IR products is provided in summary form in Appendix A. This guidance is intended to complement the SUPAC - IR guidance for industry: Immediate Release Solid Oral Dosage Forms: Scale-up and Post-Approval Changes: Chemistry, Manufacturing and Controls, In Vitro Dissolution Testing, and In Vivo Bioequivalence Documentation, with specific reference to the generation of dissolution profiles for comparative purposes.

\section{BACKGROUND}

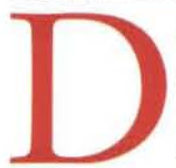

rug absorption from a solid dosage form after oral administration depends on the release of the drug substance from the drug product, the dissolution or solubilization of the drug under physiological conditions, and the permeability across the gastrointestinal tract. Because of the critical nature of the first two of these steps, in vitro dissolution may be relevant to the prediction of in vivo performance. Based on this general consideration, in vitro dissolution tests for immediate release solid oral dosage forms, such as tablets and capsules, are used to (1) assess the lot-to-lot quality of a drug product; (2) guide development of new formulations; and (3) ensure continuing product quality and performance after certain changes, such as changes in the formulation, the manufacturing process, the site of manufacture, and the scale-up of the manufacturing process.

Current knowledge about the solubility, permeability, dissolution, and pharmacokinetics of a drug product should be considered in defining dissolution test specifications for the drug approval process. This knowledge should also be used to ensure continued equivalence of the product, as well as to ensure the product's sameness under certain scale-up and postapproval changes.
New drug applications (NDAs) submitted to the Food and Drug Administration (FDA) contain bioavailability data and in vitro dissolution data, that, together with chemistry, manufacturing, and controls (CMC) data, characterize the quality and performance of the drug product. In vitro dissolution data are generally obtained from batches that have been used in pivotal clinical and/or bioavailability studies and from other human studies conducted during product development. Acceptable bioequivalence data and comparable in vitro dissolution and CMC data are required for approval of abbreviated new drug applications (ANDAs) (21 CFR 314.94). The in vitro specifications for generic products should be established based on a dissolution profile. For new drug applications, as well as generic drug applications, the dissolution specifications should be based on acceptable clinical, bioavailability, and/or bioequivalence batches.

Once the specifications are established in an $\mathrm{NDA}$, the dissolution specifications for batch-tobatch quality assurance are published in the United States Pharmacopeia (USP) as compendial standards, which become the official specifications for all subsequent IR products with the same active ingredients. In general, these compendial dissolution standards are single-point dissolution tests, not profiles.
Working Group Members:

Vinud P. Stall, ${ }^{\star \star}$ Lawrenee J. Leskn, Jim Fan, Niehalas Fleiseher, ${ }^{\star}$ James Handersen, ${ }^{\star}$ Henry Malinuwski, Mohth Makary, Iarry Duderkirk, Suva Roy, ${ }^{\star}$ Pradeep Sathe, Gurr Jai Pal Singh,

Uoyd Tillman, ${ }^{\star}$ Vi Isany ant Roger L. Williams.

These individuals currently not witb tbe FDA "To wbom corropondence should be addressed.

U.S. Department of Health and Human Services

Food and Drug Administration Center for Drug Evaluation and Research (CDER) August 1997

BP1

TABLE OF

CONTENTS

PAGE

BACKGROUND .............. I5

BIOPHARMACEUTICS

CLASSIFICATION SYSTEM . . . . 16

SETTING DISSOLUTION

SPECIFICATIONS ...........16

A. Approaches for Setting

Specifications for a New

Chemical Entity ............16

B. Approaches for Setting

Dissolution Specifications

for Generic products ........17

C. Special Cases . . . . . . . . . 17

D. Mapping or Response Surface

Methodology ............17

E. In Vivo-In Vitro Correlations ...18

F. Validation and Verification of Specifications .............18

DISSOLUTION PROFILE

COMPARISONS ............18

A. Model Independent Approach Using a Similarity Factor .......18

B. Model Independent Multivariate Confidence Region Procedure ..19

C. Model Dependent Approaches , 19 DISSOLUTION AND SUPAC-IR 19 BIOWAIVERS . . ............20

Appendix A . . . . . . . . . . . 20 REFERENCES .............22

'This guidance bas been prepared by the Immediate Release Expert Working Group of tbe Biopharmaceutics

Coordinating Committee in the Center for Drug Evaluation and Researcb (CDER) at the Food and Drug

Administration. This guidance document represents the Agency's current tbinking on the dissolution testing of immediate release solid oral dosage forms. It does not create or confer any rights for or on any person and does not operate to bind FDA or tbe public. An alternative approach may be used if such approach satisfies the requirements of the applicable statute, regulations, or botb. 


\section{FDA Immediate Release Guidance....continued}

\section{BIDPHARMACEUTICS CLASSIFICATION SYSTEM}

Based on drug solubility and permeability, the following Biopharmaceutics Classification System (BCS) is recommended in the literature (Amidon 1995):

Case 1: High Solubility - High Permeability Drugs

Case 2: Low Solubility - High Permeability Drugs

Case 3: High Solubility - Low Permeability Drugs

Case 4: Low Solubility - Low Permeability Drugs

This classification can be used as a basis for setting in vitro dissolution specifications and can also provide a basis for predicting the likelihood of achieving a successful in vivo-in vitro correlation (IVIVC). The solubility of a drug is determined by dissolving the highest unit dose of the drug in $250 \mathrm{~mL}$ of buffer adjusted between $\mathrm{pH} 1.0$ and 8.0. A drug substance is considered highly soluble when the dose/solubility volume of solution are less than or equal to 250 $\mathrm{mL}$. High-permeability drugs are generally those with an extent of absorption that is greater than $90 \%$ in the absence of documented instability in the gastrointestinal tract or those whose permeability has been determined experimentally. The BCS suggests that for high solubility, high permeability (case 1) drugs and in some instances for high solubility, low permeability (case 3 ) drugs, $85 \%$ dissolution in $0.1 \mathrm{~N} \mathrm{HCl}$ in 15 minutes can ensure that the bioavailability of the drug is not limited by dissolution. In these cases, the rate limiting step for drug absorption is gastric emptying.

The mean T50\% gastric residence (emptying) time is 15-20 minutes under fasting conditions. Based on this information, a conservative conclusion is that a drug product undergoing $85 \%$ dissolution in 15 minutes under mild dissolution test conditions in $0.1 \mathrm{~N} \mathrm{HCl}$ behaves like a solution and generally should not have any bioavailability problems. If the dissolution is slower than gastric emptying, a dissolution profile with multiple time points in multimedia is recommended.

In the case of low solubility/high permeability drugs (case 2), drug dissolution may be the rate limiting step for drug absorption and an IVIVC may be expected. A dissolution profile in multiple media is recommended for drug products in this category. In the case of high solubility/low permeability drugs (case 3), permeability is the rate controlling step and a limited IVIVC may be possible, depending on the relative rates of dissolution and intestinal transit. Drugs in case 4 (i.e., low solubility/low permeability drugs) present significant problems for oral drug delivery.

\section{SETTING DISSOLUTION SPEGIFICATIONS}

In vitro dissolution specifications are established to ensure batchto-batch consistency and to signal potential problems with in vivo bioavailability. For NDAs, the dissolution specifications should be based on acceptable clinical, pivotal bioavailability, and/or bioequivalence batches. For ANDAs/AADAs, the dissolution specifications should be based on the performance of acceptable bioequivalence batches of the drug product. The NDA dissolution specifications should be based on experience gained during the drug development process and the in vitro performance of appropriate test batches. In the case of a generic drug product, the dissolution specifications are generally the same as the reference listed drug (RLD). The specifications are confirmed by testing the dissolution performance of the generic drug product from an acceptable bioequivalence study. If the dissolution of the generic product is substantially different compared to that of the reference listed drug and the in vivo data remain acceptable, a different dissolution specification for the generic product may be set. Once a dissolution specification is set, the drug product should comply with that specification throughout its shelf life.

The International Conference on Harmonisation (ICH) Q1A guideline (Stability Testing of New Drug Substances and Drug Products) has recommended that for an NDA, three batches (two pilot and one smaller scale) be placed into stability testing. These batches also may be used to set dissolution specifications when a suitable bioequivalence relationship exists between these batches and both the pivotal clinical trial batch and the drug product intended for the market.

Three catagories of dissolution test specifications for immediate release drug products are described in the guidance.

- Single-point specifications

As a routine quality control test. (For highly soluble and rapidly dissolving drug products.)

- Two-point specifications

1. For characterizing the quality of the drug product.

2. As a routine quality control test for certain types of drug products (e.g., slow dissolving or poorly water soluble drug product like carbamazepine).

- Dissolution profile comparison

1. For accepting product sameness under SUPAC-related changes.

2. To waive bioequivalence requirements for lower strengths of a dosage form.

3. To support waivers for other bioequivalence requirements.

In the future, a two-time point approach may be useful, both to characterize a drug product and to serve as quality control specification.

\section{A. Approaches for Setting Dissolution Specifications for a New Chemical Entity}

Dissolution methodology and specifications developed by a sponsor are presented in the biopharmaceutics section (21 CFR 320.24(b)(5)), and the chemistry, manufacturing, and controls section (21 CFR 314.50(d)(1)(ii)(a)) of an NDA. The dissolution characteristics of the drug product should be developed based on consideration of the $\mathrm{pH}$ solubility profile and $\mathrm{pKa}$ of the drug substance. The drug permeability or octanol/water partition coefficient measurement may be useful in selecting the dissolution methodology and specifications. The dissolution specifications are established in consultation with 
biopharmaceutics and $\mathrm{CMC}$ review staff in the Office of Pharmaceutical Science (OPS). For NDAs, the specifications should be based on the dissolution characteristics of batches used in pivotal clinical trials and/or in confirmatory bioavailability studies. If the formulation intended for marketing differs significantly from the drug product used in pivotal clinical trials, dissolution and bioequivalence testing between the two formulations are recommended.

Dissolution testing should be carried out under mild test conditions, basket method at 50/100 rpm or paddle method at 50/75 rpm, at 15 -minute intervals, to generate a dissolution profile. For rapidly dissolving products, generation of an adequate profile sampling at 5or 10-minute intervals may be necessary. For highly soluble and rapidly dissolving drug products (BCS classes 1 and 3), a single-point dissolution test specification of NLT $85 \%(\mathrm{Q}=80 \%)$ in 60 minutes or less is sufficient as a routine quality control test for batch-to-batch uniformity. For slowly dissolving or poorly water soluble drugs (BCS class 2), a two-point dissolution specification, one at 15 minutes to include a dissolution range (a dissolution window) and the other at a later point (30, 45, or 60 minutes) to ensure $85 \%$ dissolution, is recommended to characterize the quality of the product. The product is expected to comply with dissolution specifications throughout its shelf life. If the dissolution characteristics of the drug product change with time, whether or not the specifications should be altered will depend on demonstrating bioequivalence of the changed product to the original biobatch or pivotal batch. To ensure continuous batch-tobatch equivalence of the product after scale-up and postapproval changes in the marketplace, dissolution profiles should remain comparable to those of the approved biobatch or pivotal clinical trial batch(es).

\section{B. Approaches for Setting Dissolution Specifications for Generic Products}

The approaches for setting dissolution specifications for generic products fall into three categories, depending on whether an official compendial test for the drug product exists and on the nature of the dissolution test employed for the reference listed drug. All approved new drug products should meet current USP dissolution test requirements, if they exist. The three categories are:

1. USP Drug Product Dissolution Test Available

In this instance, the quality control dissolution test is the test described in the USP. The Division of Bioequivalence, Office of Generic Drugs, also recommends taking a dissolution profile at 15minute intervals or less using the USP method for test and reference products (12 units each). The Division of Bioequivalence may also recommend submitting additional dissolution data when scientifically justified. Examples of this include (1) cases in which USP does not specify a dissolution test for all active drug substances of a combination product and (2) cases in which USP specifies use of disintegration apparatus.

2. USP Drug Product Dissolution Test Not Available; Dissolution Test for Reference Listed NDA Drug Product Publicly Available

In this instance, a dissolution profile at 15 -minute intervals of test and reference products (12 units each) using the method approved for the reference listed product is recommended. The Division of Bioequivalence may also request submission of additional dissolution testing data as a condition of approval, when scientifically justified.

3. USP Drug Product Dissolution Test Not Available; Dissolution Test for Reference Listed NDA Drug Product Not Publicly Available

In this instance, comparative dissolution testing using test and reference products under a variety of test conditions is recommended. The test conditions may include different dissolution media $(\mathrm{pH}$ 1 to 6.8), addition of surfactant, and use of apparatus 1 and 2 with varying agitation. In all cases, profiles should be generated as previously recommended. The dissolution specifications are set based on the available bioequivalence and other data.

\section{Special Cases}

1. Two-Point Dissolution Test

For poorly water soluble drug products (e.g., carbamazapine), dissolution testing at more than one time point for routine quality control is recommended to ensure in vivo product performance. Alternatively, a dissolution profile may be used for purposes of quality control.

\section{Two-Tiered Dissolution Test}

To more accurately reflect the physiologic conditions of the gastrointestinal tract, two-tiered dissolution testing in simulated gastric fluid (SGF) with and without pepsin or simulated intestinal fluid (SIF) with and without pancreatin may be employed to assess batch-to-batch product quality provided the bioequivalence is maintained.

Recent examples involving soft and hard gelatin capsules show a decrease in the dissolution profile over time either in SGF or in SIF without enzymes. This has been attributed to pellicle formation. When the dissolution of aged or slower releasing capsules was carried out in the presence of an enzyme (pepsin in SGF or pancreatin in SIF), a significant increase in the dissolution was observed. In this setting, multiple dissolution media may be necessary to adequately assess product quality.

\section{Mapping or Response Surface Methodology}

Mapping is defined as a process for determining the relationship between critical manufacturing variables $(\mathrm{CMV})$ and a response surface derived from an in vitro dissolution profile and an in vivo bioavailability data set. The CMV include changes in the formulation, process, equipment, materials, and methods for the drug product that can significantly affect in vitro dissolution (Skelly 1990, Shah 1992). The goal is to develop product specifications that will ensure bioequivalence of future batches prepared within the limits of acceptable dissolution specifications. Several experimental designs are available to study the influence of $\mathrm{CMV}$ on product performance. One approach to study and evaluate the mapping process includes (1) prepare two or more dosage formulations using CMV to study their in vitro

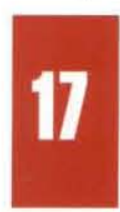




\section{FDA Immediate Release Guidance....continued}

dissolution characteristics; (2) test the products with fastest and slowest dissolution characteristics along with the standard or the to be marketed dosage form in small groups (e.g., $\mathrm{n}>12$ ) of human subjects; and (3) determine the bioavailability of the products and in vitro-in vivo relationship. The products with extreme dissolution characteristics are also referred to as side batches (Siewert 1995). If the products with the extreme range of dissolution characteristics are found to be bioequivalent to the standard or the to be marketed dosage form, future batches with dissolution characteristics between these ranges should be equivalent to one another. This approach can be viewed as verifying the limits of the dissolution specifications. Product dissolution specifications established using a mapping approach will provide maximum likelihood of ensuring stable quality and product performance. Depending on the number of products evaluated, the mapping study can provide information on in vitro-in vivo correlations and/or a rank order relationship between in vivo and in vitro data.

\section{E. In Vivo-In Vitro Correlations}

For highly water soluble (BCS classes 1 and 3) immediate release products using currently available excipients and manufacturing technology, an IVIVC may not be possible. For poorly water soluble products, BCS class 2, an IVIVC may be possible.

The value of dissolution as a quality control tool for predicting in vivo performance of a drug product is significantly enhanced if an in vitro-in vivo relationship (correlation or association) is established. The in vitro test serves as a tool to distinguish between acceptable and unacceptable drug products. Acceptable products are bioequivalent, in terms of in vivo performance, whereas unacceptable products are not. To achieve an in vitro-in vivo correlation, at least three batches that differ in the in vivo as well as the in vitro performance should be available. If the batches show differences in in vivo performance, then in vitro test conditions can be modified to correspond with the in vivo data to achieve an in vitro-in vivo correlation. If no difference is found in the in vivo performance of the batches and if the in vitro performance is different, it may be possible to modify test conditions to achieve the same dissolution performance of the batches studied in vivo. Very often, the in vitro dissolution test is found to be more sensitive and discriminating than the in vivo test. From a quality assurance point of view, a more discriminative dissolution method is preferred, because the test will indicate possible changes in the quality of the product before in vivo performance is affected.

\section{F. Validation and Verification of Specifications}

Confirmation by in vivo studies may be needed for validation of an in vitro system. In this situation, the same formulation should be used but nonformulation CMV should be varied. Two batches with different in vitro profiles should be prepared (mapping approach). These products should then be tested in vivo. If the two products show different in vivo characteristics, then the system is validated. In contrast, if there is no difference in the in vivo performance, the results can be interpreted as verifying the dissolution specification limits as discussed under mapping. Thus, either validation or verification of dissolution specifications should be confirmed.

\section{DISSOLUTION PROFILE COMPARISONS}

Until recently, single-point dissolution tests and specifications have been employed in evaluating scale-up and postapproval changes, such as (1) scale-up, (2) manufacturing site changes, (3) component and composition changes, and (4) equipment and process changes. A changed product may also be a lower strength of a previously approved drug product. In the presence of certain minor changes, the single-point dissolution test may be adequate to ensure unchanged product quality and performance. For more major changes, a dissolution profile comparison performed under identical conditions for the product before and after the change(s) is recommended (see SUPAC-IR). Dissolution profiles may be considered similar by virtue of (1) overall profile similarity and (2) similarity at every dissolution sample time point. The dissolution profile comparison may be carried out using model independent or model dependent methods.

\section{A. Model Independent Approach Using a Similarity Factor}

A simple model independent approach uses a difference factor (fl) and a similarity factor ( $\mathrm{f} 2$ ) to compare dissolution profiles (Moore 1996). The difference factor (f1) calculates the percent (\%) difference between the two curves at each time point and is a measurement of the relative error between the two curves:

$$
f_{1}=\left\{\left[\sum_{t=1}{ }^{n}\left|R_{t}-T_{t}\right|\right] /\left[\sum_{t=1}{ }^{n} R_{t}\right]\right\} \bullet 100
$$

where $\mathrm{n}$ is the number of time points, Rt is the dissolution value of the reference (prechange) batch at time $t$, and $\mathrm{Tt}$ is the dissolution value of the test (postchange) batch at time t.

The similarity factor ( $\mathrm{f} 2$ ) is a logarithmic reciprocal square root transformation of the sum of squared error and is a measurement of the similarity in the percent (\%) dissolution between the two curves.

$$
\mathrm{f}_{2}=50 \bullet \log \left\{\left[1+(1 / \mathrm{n}) \sum_{\mathrm{t}=1}{ }^{n}(\mathrm{Rt}-\mathrm{Tt})^{2}\right]^{0.5} \bullet 100\right\}
$$

A specific procedure to determine difference and similarity factors is as follows:

1. Determine the dissolution profile of two products (12 units each) of the test (postchange) and reference (prechange) products.

2. Using the mean dissolution values from both curves at each time interval, calculate the difference factor ( $\mathrm{fl}$ ) and similarity factor (f2) using the above equations.

3. For curves to be considered similar, $\mathrm{fl}$ values should be close to 0 , and $\mathrm{f} 2$ values should be close to 100 . Generally, $\mathrm{fl}$ values up to 15 $(0-15)$ and $f 2$ values greater than $50(50-100)$ ensure sameness or equivalence of the two curves and, thus, of the performance of the test (postchange) and reference (prechange) products.

This model independent method is most suitable for dissolution profile comparison when three to four or more dissolution time 
points are available. As further suggestions for the general approach, the following recommendations should also be considered:

- The dissolution measurements of the test and reference batches should be made under exactly the same conditions. The dissolution time points for both the profiles should be the same (e.g., 15, 30, 45, 60 minutes). The reference batch used should be the most recently manufactured prechange product.

- Only one measurement should be considered after $85 \%$ dissolution of both the products.

- To allow use of mean data, the percent coefficient of variation at the earlier time points (e.g., 15 minutes) should not be more than $20 \%$, and at other time points should not be more than $10 \%$.

- The mean dissolution values for Rt can be derived either from (1) last prechange (reference) batch or (2) last two or more consecutively manufactured prechange batches.

\section{B. Model Independent Multivariate Confidence Region Procedure}

In instances where within batch variation is more than $15 \%$ $\mathrm{CV}$, a multivariate model independent procedure is more suitable for dissolution profile comparison. The following steps are suggested:

1. Determine the similarity limits in terms of multivariate statistical distance (MSD) based on interbatch differences in dissolution from reference (standard approved) batches.

2. Estimate the MSD between the test and reference mean dissolutions.

3. Estimate $90 \%$ confidence interval of true MSD between test and reference batches.

4. Compare the upper limit of the confidence interval with the similarity limit. The test batch is considered similar to the reference batch if the upper limit of the confidence interval is less than or equal to the similarity limit.

\section{Model Dependent Approaches}

Several mathematical models have been described in the literature to fit dissolution profiles. To allow application of these models to comparison of dissolution profiles, the following procedures are suggested:

1. Select the most appropriate model for the dissolution profiles from the standard, prechange, approved batches. A model with no more than three parameters (such as linear, quadratic, logistic, probit, and Weibull models) is recommended.

2. Using data for the profile generated for each unit, fit the data to the most appropriate model.

3. A similarity region is set based on variation of parameters of the fitted model for test units (e.g., capsules or tablets) from the standard approved batches.

4. Calculate the MSD in model parameters between test and reference batches.

5. Estimate the $90 \%$ confidence region of the true difference between the two batches.

6. Compare the limits of the confidence region with the similarity region. If the confidence region is within the limits of the similarity region, the test batch is considered to have a similar dissolution profile to the reference batch.

\section{DISSOLUTION AND SUPAC-IR}

The SUPAC-IR guidance defines the levels of changes, recommended tests, and filing documentation to ensure product quality and performance of reference (prechange product) with postapproval changes in (1) components and composition, (2) site of manufacturing, (3) the scale of manufacturing, and (4) process and equipment changes in the manufacturing of immediate release products (FDA 1995). Depending on the level of change and the biopharmaceutics classification system of the active drug substance, the SUPAC-IR guidance recommends different levels of in vitro dissolution test and/or in vivo bioequivalence studies. Tests vary depending on therapeutic range and solubility and permeability factors of the drug substance. For formulation changes beyond those listed in the guidance, additional dissolution profile determinations in several media are recommended. For manufacturing site changes, scale-up equipment changes, and minor process changes, only dissolution testing should be sufficient to ensure unchanged product quality and performance. The SUPAC-IR guidance recommends dissolution profile comparisons for approving different

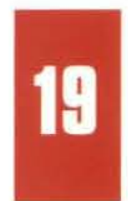




\section{FDA Immediate Release Guidance....continued}

levels of changes and documenting product sameness between the test (postchange) and reference (prechange) product. It recommends dissolution profile comparisons using a model independent approach and the similarity factor (f2).

\section{BIOWAIVERS}

In addition to routine quality control tests, comparative dissolution tests have been used to waive bioequivalence requirements (biowaivers) for lower strengths of a dosage form. For biowaivers, a dissolution profile should be generated and evaluated using one of the methods described under Section V in this guidance, "Dissolution Profile Comparisons." Biowaivers are generally provided for multiple strengths after approval of a bioequivalence study performed on one strength, using the following criteria:

For multiple strengths of IR products with linear kinetics, the bioequivalence study may be performed at the highest strength and waivers of in vivo studies may be granted on lower strengths, based on an adequate dissolution test, provided the lower strengths are proportionately similar in composition (21 CFR 320.22(d)(2)). Similar may also be interpreted to mean that the different strengths of the products are within the scope of changes permitted under the category "Components and Composition," discussed in the SUPAC-IR guidance. In all cases, the approval of additional strengths is based on dissolution profile comparisons between these additional strengths and the strength of the batch used in the pivotal bioequivalence study.

\section{Appendix A}

\section{Dissolution Testing Conditions Apparatus}

The most commonly employed dissolution test methods are (1) the basket method (Apparatus 1) and (2) the paddle method (Apparatus 2) (Shah 1989). The basket and the paddle methods are simple, robust, well standardized, and used worldwide. These methods are flexible enough to allow dissolution testing for a variety of drug products. For this reason, the official in vitro dissolution methods described in U.S. Pharmacopeia (USP), Apparatus 1 and Apparatus 2 should be used unless shown to be unsatisfactory. The in vitro dissolution procedures, such as the reciprocating cylinder (Apparatus 3) and a flow-through cell system (Apparatus 4) described in the USP, may be considered if needed. These methodologies or other alternatives/modifications should be considered on the basis of their proven superiority for a particular product. Because of the diversity of biological and formulation variables and the evolving nature of understanding in this area, different experimental modifications may need to be carried out to obtain a suitable in vivo correlation with in vitro release data. Dissolution methodologies and apparatus described in the USP can generally be used either with manual sampling or with

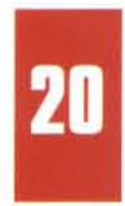
automated procedures.

\section{Dissolution Medium}

Dissolution testing should be carried out under physiological conditions, if possible. This allows interpretation of dissolution data with regard to in vivo perfor- mance of the product. However, strict adherence to the gastrointestinal environment need not be used in routine dissolution testing. The testing conditions should be based on physicochemical characteristics of the drug substance and the environmental conditions the dosage form might be exposed to after oral administration.

The volume of the dissolution medium is generally 500,900 , or $1000 \mathrm{~mL}$. Sink conditions are desirable but not mandatory. An aqueous medium with $\mathrm{pH}$ range 1.2 to 6.8 (ionic strength of buffers the same as in USP) should be used. To simulate intestinal fluid (SIF), a dissolution medium of $\mathrm{pH} 6.8$ should be employed. A higher $\mathrm{pH}$ should be justified on a case-by-case basis and, in general, should not exceed $\mathrm{pH}$ 8.0. To simulate gastric fluid (SGF), a dissolution medium of $\mathrm{pH} 1.2$ should be employed without enzymes. The need for enzymes in SGF and SIF should be evaluated on a case-by-case basis and should be justified. Recent experience with gelatin capsule products indicates the possible need for enzymes (pepsin with SGF and pancreatin with SIF) to dissolve pellicles, if formed, to permit the dissolution of the drug. Use of water as a dissolution medium also is discouraged because test conditions such as $\mathrm{pH}$ and surface tension can vary depending on the source of water and may change during the dissolution test itself, due to the influence of the active and inactive ingredients. For water insoluble or sparingly water soluble drug products, use of a surfactant such as sodium lauryl sulfate is recommended (Shah 1989, 1995). The need for and the amount of the surfactant should be justified. Use of a hydro alcoholic medium is discouraged.

All dissolution tests for IR dosage forms should be conducted at $37 \pm 0.5^{\circ} \mathrm{C}$. The basket and paddle method can be used for performing dissolution tests under multimedia conditions (e.g., the initial dissolution test can be carried out at $\mathrm{pH} 1.2$, and, after a suitable time interval, a small amount of buffer can be added to raise $\mathrm{pH}$ to 6.8). Alternatively, if addition of an enzyme is desired, it can be added after initial studies (without enzymes). Use of Apparatus 3 allows easy change of the medium. Apparatus 4 can also be adopted for a change in dissolution medium during the dissolution run.

Certain drug products and formulations are sensitive to dissolved air in the dissolution medium and will need deaeration. In general, capsule dosage forms tend to float during dissolution testing with the paddle method. In such cases, it is recommended that a few turns of a wire helix (USP) around the capsule be used.

The apparatus suitability tests should be carried out with a performance standard (i.e., calibrators) at least twice a year and after any significant equipment change or movement. However, a change from basket to paddle or vice versa may need recalibration. The equipment and dissolution methodology should include the product related operating instructions such as deaeration of the dissolution medium and use of a wire helix for capsules. Validation of automated procedures compared to the manual procedures should be well documented. Validation of determinative steps in the dissolution testing process should comply with the set standards for analytical methodology.

\section{Agitation}

In general, mild agitation conditions should be maintained during dissolution testing to allow maximum discriminating power and to 
Continued from page 20

detect products with poor in vivo performance. Using the basket method, the common agitation (or stirring speed) is $50-100 \mathrm{rpm}$; with the paddle method, it is 50-75 rpm (Shah et al., 1992). Apparatus 3 and 4 are seldom used to assess the dissolution of immediate release drug products.

\section{Validation}

Validation of the dissolution apparatus/methodology should include (1) the system suitability test using calibrators; (2) deaeration, if necessary; (3) validation between manual and automated procedures; and (4) validation of a determinative step (i.e., analytical methods employed in quantitative analysis of dissolution samples). This should include all appropriate steps and procedures of analytical methods validation.

\section{REFERENGES}

Amidon, G. L., H. Lennernas, V. P. Shab, and 7. R. Crison, 1995,

"A Theoretical Basis For a Biopharmaceutic Drug Classification:

The Correlation of In Vitro Drug Product Dissolution and In Vivo

Bioavailability," Pharmaceutical Research, 12:413-420.

FDA, 1995, Center for Drug Evaluation and Research, Guidance for Industry: Immediate Release Solid Oral Dosage Forms. Scale-up and Post-Approval Changes: Cbemistry, Mamufacturing and Controls, In Vitro Dissolution Testing, and In Vivo Bioequivalence Documentation [SUPAC-IR], November 1995.

Meyer, M. C., A. B. Straugbn, E. f. Jarvi, G. C. Wood, F. R. Pelsor, and V.P. Shah, 1992, "The Bioequivalence of Carbamazepine Tablets with a History of Climical Failures," Pharmaceutical Research, 9:1612-1616.
Moore, f. W. and H. H. Flanner, 1996, "Matbematical Comparison of Dissolution Profiles," Pbarmaceutical Teclmology, 20 (6):64-74.

Sbab, V. P., et al., 1989, "In Vitro Dissolution Profile of Water Insoluble Drug Dosage Forms in the Presence of Surfactants, " Pharmaceutical Research, 6:612-618.

Shab, V. P., et al., 1992, "Influence of Higher Rate of Agitation on Release Patterns of Immediate Release Drug Products," Fournal of Pbarmaceutical Science, 81:500-503.

Shab, V. P., 7. P. Skelly, W. H. Barr, H. Malinowski, and G. L. Amidon, 1992, "Scale-up of Controlled Release Products - Preliminary Considerations," Pharmaceutical Tecbnology, 16(5):35-40.

Sbab, V. P., et al., 1995, "In Vivo Dissolution of Sparingly Water Soluble Drug Dosage Forms," International Journal of Pharmaceutics, 125:99-106.

Siewert, M., 1995, "FIP Guidelines for Dissolution Testing of Solid Oral Products," Pbarm. Ind. 57:362-369.

Skelly, 7. P., G. L. Amidon, W. H. Barr, L. Z. Benet, 7. E. Carter, 7. R. Robinson, V. P. Shab, and A. Yacobi, 1990, "In Vitro and In Vivo Testing and Correlation for Oral Controlled/Modified-Release Dosage Forms," Pharmaceutical Research, 7:975-982.

United States Pbarmacopeia (USP), .S. Pharmacopeial Convention, Inc. Rockville, MD.

For Correspondence:

Dr. Vinod P. Shab

Office of Pbarmaceutical Science

Center for Drug Evaluation and Researcb (CDER)

Food and Drug Administration

Rockville, MD 